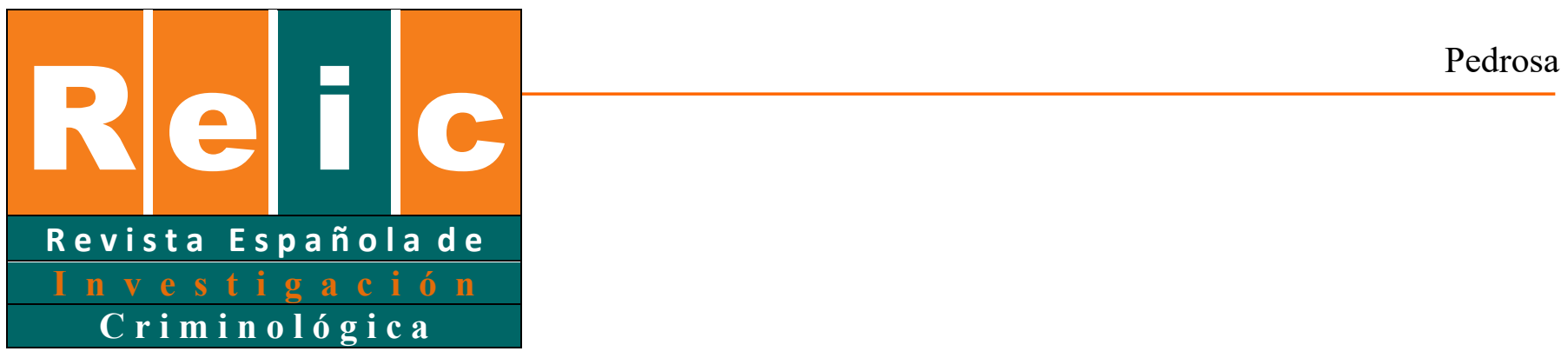

\title{
¿A quién dejamos atrás? Explorando los obstáculos de la progresión penitenciaria
}

\section{Left behind. Examining the obstacles for early release from prison}

\author{
Albert Pedrosa 1 \\ Universitat Autònoma de Barcelona
}

\section{RESUMEN}

La literatura criminológica ha mostrado cómo una salida progresiva de prisión es uno de los mejores mecanismos para lograr la reinserción. Sin embargo, los datos en el contexto español muestran que un alto porcentaje de personas terminan su condena directamente desde el segundo grado. Este trabajo propone, respecto la literatura anterior, que además de la práctica penitenciaria existen dos elementos que pueden ayudarnos a entender los obstáculos para la progresión: la experiencia del encarcelamiento y la desventaja previa. Los datos utilizados provienen del proyecto "Encarcelamiento y Reincidencia", llevado a cabo por un equipo de investigación de la Universidad Autónoma de Barcelona, a partir de cuestionarios a un total de 538 personas en la fase final de su condena en prisiones de Cataluña. Los resultados indican que la exposición a tensión durante la condena, la falta de apoyo familiar e institucional y la desventaja previa son factores que obstaculizan la progresión.

Palabras clave: prisión, salida progresiva, penalidades del encarcelamiento, apoyo social.

\footnotetext{
${ }^{1}$ La correspondencia debe dirigirse a: Albert Pedrosa Bou. Facultad de Derecho. Universitat Autònoma de Barcelona; C7de la Vall Moronta, 08193 Bellaterra (Cerdanyola del Vallès), Barcelona. Albert.pedrosa@uab.cat
}

Revista Española de Investigación Criminológica

Artículo 2, Número 17 (2019)

https://doi.org/10.46381/reic.v17i0.471

www.criminologia.net

ISSN: 1696-9219 


\begin{abstract}
Research in Criminology has shown that an early release from prison is one of the best mechanisms to achieve reintegration. However, data from the Spanish context reveal that a high percentage of people end their sentences directly from ordinary regime. This work proposes, with respect to the previous literature, that in addition to prison practice there are two elements that can help us to understand the obstacles for a progressive release: the experience of imprisonment and previous disadvantage. Data used comes from the project "Imprisonment and Recidivism" carried out by a research team of the Universitat Autònoma of Barcelona, based from a sample of 538 questionnaires of inmates in the final period of their sentences in prisons of Catalonia. Results indicate that exposure to strain during the sentence, lack of family and institutional support and previous disadvantage are factors that difficult a progressive release from prison.
\end{abstract}

Keywords: prison, early release, pains of imprisonment, social support.

\title{
1. Introducción
}

De entre las distintas finalidades de la pena de prisión la legislación española establece que esta se orientará hacia la reeducación y reinserción social de los penados, finalidad que se debería lograr durante la misma condena (Cid, 2005). Uno de los métodos más idóneos para conseguirlo es mediante una salida progresiva hacia la comunidad (ver la revisión realizada por Cid \& Tébar, 2010) puesto que esta reducirá la reincidencia (Luque, Ferrer \& Capdevila, 2005) y, además, permitirá al sujeto prepararse para su vida en libertad, acceder a mecanismos de supervisión en la comunidad menos coercitivos, y restablecer o mantener vínculos con instituciones clave como el mercado de trabajo o las relaciones familiares prosociales. Con este fin, el modelo de tratamiento y reinserción español se estructura alrededor de la idea de progresión donde el penado, mediante su comportamiento y realización del tratamiento según sus necesidades, irá accediendo a condiciones de semilibertad antes de la finalización de su condena (art. 65.2 Ley Orgánica General Penitenciaria). Sin embargo, los datos disponibles muestran una realidad que dista de este modelo ideal, ya que encontramos un alto porcentaje de personas que finalizan su condena directamente en segundo grado y sin acceso a condiciones de semilibertad, siendo esta la forma más común de retorno a la comunidad (Cid \& Tébar, 2010; Ibàñez \& Cid, 2016), lo que hace dudar de si estamos ante un verdadero sistema progresivo. Estos obstáculos parecen

Revista Española de Investigación Criminológica

Artículo 2, Número 17 (2019)

https://doi.org/10.46381/reic.v17i0.471

www.criminologia.net

ISSN: 1696-9219 
ser mayores para el acceso a la libertad condicional, especialmente para aquellas personas con un pronóstico de riesgo elevado (Cid \& Tébar, 2010).

Este trabajo pretende analizar la progresión dentro de prisión partiendo de la siguiente pregunta: ¿Quién se queda atrás en nuestro sistema penitenciario? En este sentido, se establece como relevante conocer qué factores influyen en que una persona pueda finalizar su condena con una salida progresiva hacia la comunidad, puesto que aquellos que se quedan atrás no sólo reincidirán en mayor medida (Luque et al. 2005), sino que además quedarán más expuestos a los males de la pena y a sus consecuencias más negativas.

Los estudios que han tratado de analizar y dar explicación a este fenómeno han destacado determinados factores relativos al historial delictivo y a la trayectoria penitenciaria de la persona como aquellos que más obstaculizan una salida progresiva (Cid \& Tébar, 2014; Tébar, 2004). Sin embargo, y tal como ocurre en el estudio de la violencia en prisión, estos estudios no suelen contar con una base teórica que haga referencia a las teorías criminológicas (Blevins, Listwan, Cullen \& Jonson, 2010), y su enfoque se suele centrar en la práctica penitenciaria, siendo difícil en ocasiones interpretar el mecanismo causal que favorece u obstaculiza la progresión. Al respecto, en este trabajo trataré de aportar evidencia acerca de los mecanismos que pueden vincular dos elementos centrales, la experiencia del encarcelamiento y la desventaja de los individuos, con la progresión penitenciaria. El cómo las personas viven su condena resulta relevante puesto que aquellas más expuestas a fuentes de tensión pueden experimentar emociones negativas y mal comportamiento, desvinculándose del tratamiento y viéndose excluidas de la progresión. Este efecto negativo sería aún mayor si la persona no recibe apoyo y no cuenta con recursos para afrontar estas experiencias negativas. Por su parte, la desventaja previa nos ayudará a entender por qué algunos individuos viven peor su condena y son más estigmatizados dentro de los centros. Es por ello que aquí se propone que conceptos incluidos en las teorías de la tensión, del control y del etiquetamiento pueden ser útiles y relevantes para el análisis de este fenómeno a partir del estudio de los efectos de la prisión sobre los individuos y su adaptación y relación con la institución penitenciaria, ayudándonos también a interpretar los resultados de la literatura previa.

Revista Española de Investigación Criminológica

Artículo 2, Número 17 (2019)

https://doi.org/10.46381/reic.v17i0.471

www.criminologia.net

ISSN: 1696-9219 
Este artículo tiene un doble objetivo: por un lado, pretende incrementar el conocimiento sobre qué individuos progresan durante su condena y cuáles no; y por otro, pretende mostrar la relevancia de determinadas teorías criminológicas para mejorar nuestra comprensión de este fenómeno.

\section{Marco teórico}

\subsection{La progresión penitenciaria}

Un análisis de la literatura previa muestra que contamos con un número limitado de estudios que hayan tratado de identificar las causas y obstáculos de la progresión. Los estudios disponibles que han tratado aspectos relacionados con este proceso y sus distintas fases se han centrado en: i) la concesión de la libertad condicional; ii) la revocación del régimen abierto; iii) la clasificación inicial en tercer grado.

Sobre la primera cuestión, la única investigación que ha tratado de determinar qué variables aumentan la probabilidad de finalizar la condena en libertad condicional es la llevada a cabo por Tébar (2004), quien analizó dos muestras de individuos en la fase final de su condena, una compuesta por individuos en libertad condicional y otra por personas que no se encontraban en esta situación. La investigación concluye que aquellas personas más sancionadas y con un historial delictivo previo tienen menor probabilidad de finalizar su condena en libertad condicional, mientras que aquellas que han pasado menos tiempo en prisión preventiva y que han tenido más días de salida tienen una probabilidad mayor.

En segundo lugar, Cid y Tébar (2014) analizaron datos de una muestra de personas que tuvieron una revocación en el periodo de un año para explorar las causas de la regresión de grado y cómo esta puede suponer un obstáculo para la progresión. Los autores concluyeron que las personas regresadas en general tienden a finalizar la condena sin volver a acceder al régimen abierto. Aunque sus datos no permiten afirmar qué factores individuales pueden afectar a dicho proceso, los autores ponen el énfasis en causas inherentes al sistema, como los tiempos para otorgar permisos y decidir sobre nuevas clasificaciones (Cid \& Tébar, 2014). En una línea parecida, un estudio de Férez-Mangas y Andrés-Pueyo (2015) analizó qué

\section{Revista Española de Investigación Criminológica}

Artículo 2, Número 17 (2019)

https://doi.org/10.46381/reic.v17i0.471

www.criminologia.net

ISSN: 1696-9219 
factores permiten predecir el quebrantamiento de los permisos penitenciarios, llegando a la conclusión que el mal comportamiento en prisión y la falta de apoyo social eran los elementos más relevantes al respecto.

En tercer lugar, Capdevila, Parés, Ferrer, Luque y Torrecillas (2006) estudiaron una muestra compuesta por todas las personas inicialmente clasificadas en tercer grado en el periodo de un año en Cataluña con el objetivo de observar las diferencias respecto de aquellas clasificadas inicialmente en régimen ordinario. Estos autores comprobaron que las personas clasificadas inicialmente en tercer grado presentaban un perfil socioeconómico más estable y normalizado. En cambio, los autores encontraron diferencias mínimas en el tipo de delitos cometidos o la trayectoria penal respecto de los clasificados inicialmente en segundo grado. Esto es importante, ya que parecería que en la decisión de clasificación inicial pesa más la trayectoria previa de los individuos que el comportamiento actual, hecho que se discutirá más adelante.

Los estudios anteriormente descritos se han centrado en el análisis de datos oficiales, pero también es necesario conocer la perspectiva de los profesionales y saber si estos identifican los mismos obstáculos en la práctica penitenciaria. En este sentido, Ibàñez y Cid (2016) entrevistaron a profesionales del medio penitenciario para conocer cómo estos afrontan el proceso de reinserción de las personas que finalizan en segundo grado y los factores que dificultan la progresión. Los autores señalan cuatro elementos que de acuerdo con los profesionales obstaculizan este proceso: tener nacionalidad extranjera, tener expedientes disciplinarios, incumplir el programa de tratamiento, y no pagar la responsabilidad civil. Por lo tanto, vemos como los profesionales también identifican algunos factores asociados a la práctica penitenciaria y a la trayectoria de los individuos dentro de prisión como obstáculos para la progresión. 


\subsection{La experiencia del encarcelamiento}

La primera de las dimensiones que este trabajo propone para el estudio de la progresión es la vivencia de la condena y cómo esta afecta al comportamiento de los individuos. La importancia del ambiente en prisión y su influencia sobre la persona penada fue tratada en el clásico texto de Sykes (1958) “The society of captives”, donde dicho autor advertía que el encarcelamiento y los males que este comporta -las llamadas penalidades del encarcelamiento- podían generar una respuesta violenta o antisocial en los individuos, en tanto se adaptarían a dichos males adoptando una contracultura opuesta a la institución carcelaria.

Más recientemente estas ideas han sido incorporadas por los teóricos de la tensión, bajo el paraguas de la Teoría General de la Tensión (en adelante GST, por sus siglas en inglés) propuesta por Robert Agnew (1992), quien propone que el comportamiento transgresor de los individuos es consecuencia de la exposición a estímulos negativos o a la pérdida de estímulos positivos, lo cual comportará la experimentación de emociones negativas tales como la ira o la frustración. Ante dichas emociones negativas, los sujetos podrán adaptarse y hacerles frente mediante diversas estrategias y comportamientos, incluyendo la violencia como forma de adaptación a la tensión (Agnew, 1992, 2001, 2006). Relacionando esto con el concepto de penalidades del encarcelamiento, Listwan, Sullivan, Agnew, Cullen y Colvin (2013) aplican los principios de la GST para explorar su impacto en la reincidencia, concluyendo que formas específicas de tensión, especialmente el ambiente negativo en prisión y las relaciones negativas con otros internos, contribuyen a una mayor reincidencia. Por lo tanto, estos resultados apoyarían la literatura que ha puesto de manifiesto que el encarcelamiento puede ser criminógeno (Cid, 2009; Nagin, Cullen \& Jonson, 2009; entre otros) en comparación con otras penas que suponen un coste emocional y social menor para el individuo. Así, contamos con evidencia de que la exposición a tensión se relaciona con un peor comportamiento en prisión (Steiner, Butler \& Ellison, 2014) y que esta puede generarse a partir de la exposición a situaciones negativas, como la victimización en prisión

Revista Española de Investigación Criminológica

Artículo 2, Número 17 (2019)

https://doi.org/10.46381/reic.v17i0.471

www.criminologia.net

ISSN: 1696-9219 
o la exclusión en la obtención de beneficios, incluyendo la no obtención de una liberación anticipada (Blevins et al. 2010).

Un elemento clave de la GST es cómo las personas hacen frente a la tensión a la que se ven expuestas. En este sentido, los recursos y capital social con los que cuenta la persona serán claves para entender cómo los individuos se adaptan a la tensión de una forma prosocial o anti-normativa. Cobra importancia, por lo tanto, el concepto de apoyo social, tanto informal como formal, el cual puede ser moderador de la tensión (Cullen, 1994). En esta línea Colvin, Cullen y VanderVen (2002) adoptan una perspectiva integrada que incluye la coerción como elemento que lleva al delito, y el apoyo social como factor de protección frente a este. Aplicado al estudio del desorden en prisión, Colvin (2007) propone que el uso de métodos coercitivos por parte del personal aumenta la percepción de ilegitimidad y el mal comportamiento, mientras que, si la institución da apoyo a través del trabajo, el tratamiento y mediante sus profesionales, esto incrementará la vinculación de los penados con el cumplimento del régimen penitenciario. Esto es relevante en tanto la prisión es una institución que puede coercionar a las personas encarceladas mediante múltiples mecanismos, especialmente a través de las sanciones y castigos dentro del centro.

Por otra parte, menor atención se ha prestado a la importancia de los mecanismos de afrontamiento de la tensión a nivel individual, si bien sabemos que estos son un elemento que influye en las diferencias entre individuos a la hora de adaptarse a la tensión de una forma delictiva o no (Broidy \& Agnew, 1997). La literatura sobre esta cuestión se ha centrado especialmente en el papel de la autoestima en la relación tensión-delincuencia, encontrando cierta evidencia de que la autoestima puede ser un factor de afrontamiento frente a la tensión (Kort-Butler, 2010). Por otro lado, la autoestima se relaciona con unas expectativas más positivas al final de la condena (Visher \& O'Connell, 2012) y, por lo tanto, con una menor anticipación de fuentes de tensión. Este trabajo incluirá estos aspectos en un intento de aportar conocimiento sobre dicha cuestión.

En síntesis, la experiencia de la condena puede ser relevante porque aquellas personas más expuestas a fuentes de tensión y coerción, tales como un ambiente negativo, victimización o ser sancionado, experimentarán en mayor medida sentimientos negativos.

\section{Revista Española de Investigación Criminológica}

Artículo 2, Número 17 (2019)

https://doi.org/10.46381/reic.v17i0.471

www.criminologia.net

ISSN: 1696-9219 
Estos sentimientos negativos no solo generarán un peor estado emocional en los individuos, sino que además se podrán traducir en un peor comportamiento en prisión, conductas de desafío para hacer frente a la percepción de ilegitimidad, y una desvinculación de la institución penitenciaria y su personal. Todos estos elementos, por lo tanto, resultarían en obstáculos para una progresión efectiva. Ante esto, el apoyo social y por parte de los profesionales puede ser un factor protector clave que ayude a las personas a adaptarse y hacer frente a los males de la pena, favoreciendo sus posibilidades de progresar.

\subsection{La desventaja previa}

El concepto de desventaja es otra de las grandes dimensiones que, según mi propuesta, puede ser relevante para el estudio de la progresión. Para entender el rol de las instituciones de control en las trayectorias delictivas, Sampson y Laub (1997) propusieron la teoría de la Desventaja Acumulada a partir de la integración de la teoría del Control y del Etiquetamiento. Los autores proponen que aquellos individuos que en la juventud cometen un acto delictivo pueden verse expuestos a una reacción social estigmatizadora y dicha etiqueta, tal y como proponen Link, Cullen, Struening, Shrout y Dohrenwend (1989), comportará una serie de consecuencias para los individuos relacionadas con su exclusión de diversas instituciones sociales. Aplicada al estudio del delito, estos jóvenes etiquetados tendrán peores relaciones y control familiar, se desvincularán de la escuela (Sampson \& Laub 1997), y a la larga se verán excluidos del mercado de trabajo (Nilsson, Bäckman \& Estrada, 2013). Este proceso es acumulativo en tanto estas exclusiones reducirán el control formal e informal sobre el individuo, llevándolo a cometer nuevos delitos que a su vez generarán nuevos procesos de etiquetamiento y exclusión, como si de una bola de nieve se tratase (Sampson \& Laub, 1997). Este trabajo parte de la base de que estos procesos de exclusión que se dan en otras instituciones de nuestra sociedad tendrán una continuidad y se producirán también en el espacio de la prisión. Esto es especialmente interesante porque, de hecho, esta idea de continuidad también está presente en las teorías de la tensión. En el marco de la GST, Agnew (2001, 2009) habla de los efectos acumulativos ante una exposición continuada a la tensión,

Revista Española de Investigación Criminológica

Artículo 2, Número 17 (2019)

https://doi.org/10.46381/reic.v17i0.471

www.criminologia.net

ISSN: 1696-9219 
entre los que destaca el desarrollo de emociones negativas o una disminución del control; y de la misma forma, Colvin et al. (2002) también destacan que la coerción y la falta de apoyo pueden generar nueva coerción en el futuro.

Este planteamiento nos puede ayudar a entender qué personas serán más vulnerables a los efectos de la tensión a dos niveles: a nivel individual, los efectos del proceso de etiquetamiento harán al individuo más vulnerable a sufrir nuevas consecuencias negativas en el futuro, entre las que se encuentran una disminución de la autoestima y de su red social (Link et al. 1989), así como una exclusión de las actividades convencionales, lo cual puede llevar a la búsqueda de apoyo en grupos anti-normativos (Sampson \& Laub, 1997). Por otro lado, esta teoría nos permite incorporar la importancia de la posición en la estructura social de los individuos, puesto que aquellos que se vean más excluidos de las distintas instituciones relevantes de nuestra sociedad, especialmente la formativa y laboral, arrastrarán consigo esa exclusión que también se reproducirá dentro de prisión y en el futuro, generando barreras para la reinserción (Laub \& Sampson, 1993). Aunque en el contexto español contamos con poca evidencia sobre estas cuestiones, sí sabemos que las personas reincidentes suelen presentar mayores características de desventaja, especialmente en el ámbito laboral (Capdevila et al. 2015). Cabe plantear, pues, si la trayectoria previa de los individuos tendrá una influencia en cómo estos vivan su condena y se adapten a prisión.

\section{Hipótesis}

En apartados anteriores se han presentado algunos conceptos que, a mi juicio, pueden ofrecer un marco explicativo de referencia para entender el comportamiento en prisión, los efectos de la pena sobre los individuos y, por lo tanto, qué individuos tendrán mayor probabilidad de progresar. Alrededor de estas ideas, experiencia del encarcelamiento, adaptación a él y desventaja, se estructurarán las variables empleadas en el análisis. Con el objetivo de conocer la capacidad explicativa de dicho marco teórico el artículo tratará de explorar las siguientes hipótesis, vinculadas a los conceptos anteriores:

H1: Una mayor exposición a tensión durante el encarcelamiento dificultará la progresión.

Revista Española de Investigación Criminológica

Artículo 2, Número 17 (2019)

https://doi.org/10.46381/reic.v17i0.471

www.criminologia.net

ISSN: 1696-9219 
H2: El apoyo social aumentará la probabilidad de progresar.

H3: Una trayectoria de mayor desventaja dificultará la progresión.

\section{Método}

\subsection{Muestra}

Los datos utilizados provienen del proyecto Encarcelamiento y Reincidencia (DER201455315-P) financiado por el Ministerio de Economía y Competitividad, y llevado a cabo por un equipo de investigación de la Universitat Autònoma de Barcelona, del cual el autor del presente artículo forma parte. Este proyecto cuenta con una muestra de personas que entre 2016 y 2017 se encontraban en la fase final de su condena (últimos 4 meses) en instituciones penitenciarias de Cataluña o en libertad condicional. Las personas elegibles para la muestra eran todas aquellas que en el periodo descrito se encontrasen en la fase final de su condena, con el objetivo de que esta fuera representativa de toda la población que en un año finalizara su condena en Cataluña. En total, se obtuvo una lista de 1.394 individuos, y de estos se contactó con 1.072 personas (elegibles), en los centros o fuera de ellos, para explicarles el objetivo del proyecto y pedir su colaboración. Del total de personas elegibles finalmente 538 personas accedieron a participar, lo que supone una tasa de respuesta del 50.2\%. En la tabla 1 se pueden observar algunos descriptores de las características de nuestra muestra, comparándolas con los datos de la población general ${ }^{2}$.

\footnotetext{
${ }^{2}$ Hay una sobrerrepresentación de personas clasificadas en segundo grado, viéndose infrarrepresentadas las personas en LC, debido a la dificultad de contacto con estas. 
Tabla 1.

Comparación entre la muestra y la población

\begin{tabular}{lccccc}
\hline & \multicolumn{2}{c}{ Población } & \multicolumn{2}{c}{ Muestra } & Diferencia \\
\cline { 2 - 4 } & $\mathbf{N}$ & $\mathbf{\%}$ & $\mathbf{N}$ & $\mathbf{\%}$ & \% \\
\hline Sexo & & & & & \\
$\quad$ Hombres & 2834 & 92.0 & 488 & 90.9 & -1.1 \\
$\quad$ Mujeres & 247 & 8 & 50 & 9.1 & 1.1 \\
$\quad$ Edad & & & & & \\
$\quad$ Hasta 35 años & 1237 & 40.1 & 206 & 38.3 & -1.8 \\
$\quad$ Más de 35 años & 1844 & 59.9 & 332 & 61.7 & 1.8 \\
$\quad$ Nacionalidad & 1842 & 59.8 & 328 & 61.0 & 1.2 \\
$\quad$ Españoles & 1239 & 40.2 & 210 & 39.0 & -1.2 \\
$\quad$ Extranjeros & & & & & \\
$\quad$ Finalización de la condena & 1737 & 56.4 & 337 & 62.6 & 6.2 \\
$\quad$ Primer y segundo grado & 566 & 18.4 & 100 & 18.6 & 0.2 \\
$\quad$ Tercer grado & 778 & 25.3 & 101 & 18.8 & -6.5 \\
$\quad$ Libertad condicional & 3081 & 100 & 538 & 100 & \\
\hline TOTAL & & & & & \\
\hline
\end{tabular}

*Total de las personas que finalizaron su condena en 2016 en centros de Cataluña. Datos facilitados por la Dirección general de Servicios Penitenciarios de Cataluña.

\subsection{Instrumentos y medidas}

Los datos se han obtenido a partir de un cuestionario empleado en el proyecto Returning Home (Visher \& O’Connell, 2012), el cual fue adaptado a nuestro contexto por el equipo investigador. De forma adicional, se añadieron nuevas preguntas y escalas sobre conceptos que la investigación previa ha demostrado relevantes. El cuestionario final cuenta con 270 preguntas, que tratan diversos aspectos relacionados con la trayectoria de los individuos, su vivencia de la condena y sus perspectivas de futuro. Previo consentimiento, los participantes respondieron individualmente al cuestionario, recibiendo asistencia por parte del equipo investigador cuando fuera necesario. Los cuestionarios tenían una duración media de 75 minutos y los participantes fueron gratificados con la cantidad de $10 €$.

Se ha generado un modelo de regresión logística para explorar las hipótesis planteadas. En la tabla 2 se pueden observar los descriptivos de las diferentes variables incluidas en el modelo, las cuales se comentarán a continuación: 


\section{Tabla 2.}

Análisis descriptivos

\begin{tabular}{|c|c|c|c|c|}
\hline & Mínimo & Máximo & Media & $\begin{array}{c}\text { Desviación } \\
\text { estándar }\end{array}$ \\
\hline \multicolumn{5}{|l|}{ Variable Dependiente } \\
\hline Salida progresiva & 0 & 1 & .37 & .484 \\
\hline \multicolumn{5}{|l|}{ Variables de Control } \\
\hline Hombre & 0 & 1 & .91 & .291 \\
\hline Edad & 19 & 76 & 39.77 & 10.98 \\
\hline Extranjero & 0 & 1 & .39 & .488 \\
\hline Meses de condena & 2 & 504 & 43.90 & 44.32 \\
\hline Condena por delito violento & 0 & 1 & .54 & .499 \\
\hline Participación en el tratamiento & 0 & 1 & .877 & .328 \\
\hline Trabajo durante la condena & 0 & 1 & .75 & .436 \\
\hline Revocación del tercer grado & 0 & 1 & .21 & .407 \\
\hline Responsabilidad Civil & 0 & 1 & .555 & .497 \\
\hline \multicolumn{5}{|c|}{$\begin{array}{l}\text { Variables independientes (Experiencia del } \\
\text { encarcelamiento y apoyo social) }\end{array}$} \\
\hline Sanciones frecuentes & 0 & 1 & .13 & .340 \\
\hline Autoestima & 1.3 & 4 & 3.059 & .560 \\
\hline Trato legítimo & 1 & 4 & 2.30 & .727 \\
\hline Ambiente en prisión & 1 & 4 & 2.543 & .723 \\
\hline Victimización en prisión & 1 & 4 & 1.48 & .635 \\
\hline Optimismo & 1 & 4 & 3.337 & .556 \\
\hline Apoyo familiar & 1 & 4 & 3.105 & .920 \\
\hline Apoyo profesional & 0 & 1 & .65 & .476 \\
\hline \multicolumn{5}{|c|}{ Variables independientes (Desventaja) } \\
\hline Reincidente & 0 & 1 & .461 & .498 \\
\hline Frecuencia consumo de drogas & 2 & 10 & 3.34 & 1.548 \\
\hline Desventaja educativa & 0 & 2 & .58 & .757 \\
\hline Meses cotizados & 0 & 564 & 87.79 & 103.792 \\
\hline No tiene lugar de para vivir & 0 & 1 & .08 & .271 \\
\hline
\end{tabular}




\subsection{Variable dependiente}

La variable dependiente del modelo es la salida progresiva, variable dicotómica que diferencia aquellas personas que en los cuatro meses previos a la finalización de su condena se encontrasen en primer o segundo grado (0) de aquellos que finalizaron su condena en medio abierto, ya fuera en tercer grado o libertad condicional (1).

\subsection{Variables de control}

Dentro de las variables de control se han considerado, por un lado, variables sociodemográficas, y por otro, las variables que la literatura previa ha mostrado como importantes en la explicación de la progresión. Como variables sociodemográficas se han incluido el sexo y la edad. En cuanto a las características de la condena que pueden obstaculizar la progresión se incluyen los meses de condena, si la persona tuvo o no una revocación del tercer grado, si tuvo responsabilidad civil, y si fue condenada por delito violento. Por último, se incluye una variable que distingue entre personas con nacionalidad española y personas extranjeras. Para medir la participación en el tratamiento se han incluido dos variables dicotómicas, la primera en referencia a si la persona ha trabajado durante la condena, y una segunda para diferenciar aquellas personas que no han participado en ningún programa o actividad de tratamiento de aquellas que sí3

\footnotetext{
${ }^{3}$ Se realizaron modelos alternativos con otras variables como la valoración del tratamiento, pero los resultados no variaban sustancialmente. Se optó por emplear esta variable debido a que es la que comportaba menos casos perdidos.
} 


\subsection{Variables independientes}

\section{Experiencia del encarcelamiento y apoyo social}

En primer lugar, se han incluido algunas de las variables propuestas por Listwan et al. (2013) como factores generadores de tensión en prisión. Se incluye una escala de ambiente negativo (alpha $=.87)$, ya utilizada por estos autores, y que pregunta por la percepción sobre las relaciones entre internos y funcionarios en el centro o la percepción de seguridad, entre otras cuestiones. También se incluye una escala de optimismo al final de la condena (alpha =.87), empleada por Visher y O'Connell (2012), acerca de la probabilidad de sufrir determinados problemas una vez que se retorne a la comunidad, variable que nos sirve para identificar fuentes de tensión anticipada. Finalmente, se ha creado una escala de victimización sufrida en prisión por parte de otros internos (alpha =.79). En segundo lugar, se incluyen dos variables relacionadas con la coerción, una escala de percepción de legitimidad acerca del trato recibido por parte de los funcionarios (alpha $=.88)$, y una segunda variable dicotómica que distingue entre aquellos individuos que no han sido sancionados, o lo han sido de forma ocasional durante la condena, y aquellos con sanciones frecuentes. Finalmente, para explorar la importancia del estado emocional del sujeto en el afrontamiento de la tensión, se incluye una escala de autoestima $($ alpha $=.60)$, escala utilizada previamente en Visher y O'Connell (2012).

En relación con el apoyo recibido se han tenido en cuenta tanto las fuentes informales como formales. En referencia a las primeras, se creó una escala de apoyo familiar convencional (alpha $=.86$ ) que incluía tanto apoyo emocional como instrumental. En cuanto al apoyo profesional se preguntó a las personas si estas habían tenido o no a alguien del personal que se hubiera preocupado de forma significativa por su proceso de reinserción y su situación durante la condena.

\section{Variables de desventaja}

Se han incluido variables relativas a los ámbitos de desventaja que la literatura ha mostrado más relacionados con el delito y la exclusión como son la desventaja penal, educativa, laboral y la vivienda. Para ello se han tomado en cuenta las siguientes variables: si la persona tenía

Revista Española de Investigación Criminológica

Artículo 2, Número 17 (2019)

https://doi.org/10.46381/reic.v17i0.471

www.criminologia.net

ISSN: 1696-9219 
condenas previas, una escala de frecuencia de consumo de drogas previo a la entrada a prisión ${ }^{4}$, los meses cotizados a la seguridad social, una variable dicotómica para saber si la persona cuenta con lugar para vivir una vez finalice su condena, y finalmente y para medir la desventaja educativa, un índice que agrupa dos variables dicotómicas según si la persona ha alcanzado la educación obligatoria en el momento actual y si a los 14 años asistía regularmente a la escuela.

\section{Resultados}

A nivel bivariado todas las variables propuestas para la exploración de las hipótesis correlacionan significativamente con la variable dependiente y en el sentido esperado, observándose un mayor efecto por parte de aquellas variables de coerción y las de desventaja.

En la tabla 3 se incluyen los resultados del modelo de regresión logística propuesto. El modelo es significativo, con un Chi-cuadrado de 219.030 y con una pseudo-R2 de .503. El ajuste del modelo, por lo tanto, es óptimo y significativo, permitiendo clasificar (en base a la variable dependiente) correctamente 80 de cada 100 casos. Debido a la pérdida de información en algunas variables en el modelo final se han incluido 472 casos.

En primer lugar, se ha llevado a cabo un modelo alternativo únicamente con las variables de control (no incluido aquí) observando un descenso en el ajuste del modelo (.190) y donde únicamente se clasificaban correctamente 69 de cada 100 casos. Por lo tanto, podemos afirmar que el hecho de incorporar las variables propuestas por nuestro marco teórico hace que el ajuste y la predicción del modelo mejoren significativa y sustancialmente, por lo que estas variables se presentan como adecuadas para la explicación de la progresión.

\footnotetext{
${ }^{4}$ Incluyendo consumo de alcohol, heroína, cocaína, anfetaminas, éxtasis, hachís, psicofármacos, y otras drogas ilegales.
}

Revista Española de Investigación Criminológica

Artículo 2, Número 17 (2019)

https://doi.org/10.46381/reic.v17i0.471

www.criminologia.net

ISSN: 1696-9219 


\section{Tabla 3.}

Resultados del modelo de regresión logística

\begin{tabular}{|c|c|c|c|c|}
\hline Variables & B & SE & Sig. & $\operatorname{Exp}(B)$ \\
\hline \multicolumn{5}{|l|}{ Variables de Control } \\
\hline Hombre & -.124 & .430 & .773 & .883 \\
\hline Edad & -.006 & .016 & .693 & .994 \\
\hline Extranjero & -.697 & .299 & .020 & .498 \\
\hline Meses de condena & .016 & .004 & .000 & 1.016 \\
\hline Condena por delito violento & -.681 & .277 & .014 & .506 \\
\hline Ha participado en tratamiento & -.762 & .421 & .071 & .467 \\
\hline Ha trabajado en la condena & -.444 & .306 & .146 & .641 \\
\hline Revocación del tercer grado & -1.144 & .339 & .001 & .319 \\
\hline Responsabilidad Civil & -.399 & .269 & .138 & .671 \\
\hline \multicolumn{5}{|c|}{$\begin{array}{l}\text { Variables independientes (Experiencia del } \\
\text { encarcelamiento y apoyo social) }\end{array}$} \\
\hline Sanciones frecuentes & -1.914 & .611 & .002 & .147 \\
\hline Autoestima & .935 & .268 & .000 & 2.547 \\
\hline Trato legítimo & .416 & .174 & .017 & 1.515 \\
\hline Ambiente en prisión & .406 & .227 & .075 & 1.500 \\
\hline Victimización en prisión & -.582 & .229 & .011 & .559 \\
\hline Optimismo & -.529 & .283 & .062 & .589 \\
\hline Apoyo familiar & .326 & .150 & .029 & 1.386 \\
\hline Apoyo profesional & .863 & .278 & .002 & 2.370 \\
\hline \multicolumn{5}{|c|}{ Variables independientes (Desventaja) } \\
\hline Reincidente & -.753 & .265 & .005 & .471 \\
\hline Frecuencia consumo de drogas & -.311 & .110 & .005 & .732 \\
\hline Desventaja educativa & -.174 & .182 & .337 & .840 \\
\hline Meses cotizados & .004 & .002 & .018 & 1.004 \\
\hline No tiene lugar de para vivir & -.852 & .548 & .120 & .427 \\
\hline Constante & -1.514 & 1.659 & .361 & .220 \\
\hline
\end{tabular}

Si observamos con detalle los resultados, en primer lugar, observamos como algunas de las variables relacionadas con la práctica y trayectoria penitenciaria que la literatura anterior destacaba como importantes también lo son en nuestro modelo. Así pues, la condición de extranjería, la duración de la condena, haber cometido un delito violento, y haber sufrido una revocación de grado, influyen en la progresión. Destaca especialmente que las personas con nacionalidad extranjera presentan, cuando el resto de variables se mantienen constantes, la mitad de probabilidades de salir de forma progresiva que las personas españolas. Aquellas personas que han sido condenadas por un delito violento tienen la mitad 
de probabilidades de progresar, mientras que haber sufrido una revocación de grado las reduce en un $68 \%$. Por otra parte, la duración de la condena se relaciona significativamente con el tipo de salida. El sentido de la asociación indica que cuanto mayor duración tiene la condena, mayor probabilidad de finalizar de forma progresiva. En cambio, contrariamente a lo esperado el hecho de tener responsabilidad civil, haber trabajado durante la condena, o no haber seguido ningún tipo de tratamiento no parece influir en la progresión. Las variables sexo y edad tampoco han resultado significativas.

En cuanto a la primera hipótesis planteada, vemos como solo algunas de las fuentes de tensión y coerción identificadas se relacionan con nuestra variable dependiente. Por una parte, aquellas personas que hayan sido más victimizadas en prisión tendrán mayores dificultades para progresar, siendo el efecto de esta asociación importante. Por otra, aquellas personas que se han sentido tratadas con mayor legitimidad y aquellas con una mayor autoestima saldrán en mayor medida de forma progresiva, siendo destacable el efecto de esta última variable. También observamos que haber sido sancionado con frecuencia se asocia negativamente con una salida progresiva, con un efecto considerable. Por el contrario, la tensión anticipada y el ambiente en prisión no resultan significativos.

En referencia a la segunda hipótesis, apreciamos como se confirma que el apoyo familiar y el apoyo formal ofrecido por la institución penitenciaria a través de una figura de referencia son variables significativas. Ambos tipos de apoyo muestran una asociación positiva con la progresión, siendo destacable el efecto del apoyo de los profesionales.

En relación con la última hipótesis planteada, observamos que ser reincidente y tener un historial previo de consumo de drogas se relacionan con la progresión, mientras que de entre las variables de desventaja socioeconómica solo la desventaja laboral es significativa. Así, las personas reincidentes tienen un 53\% menos de probabilidades de finalizar su condena actual de forma progresiva, mientras que cada unidad de aumento en la escala de consumo previo supone una reducción del 26\% de la probabilidad de progresión. Si atendemos al historial laboral previo, vemos como a medida que este aumenta también lo hacen las probabilidades de finalizar la condena en régimen abierto. Por su parte, ni la desventaja educativa ni la falta de vivienda resultan significativas.

\section{Revista Española de Investigación Criminológica}

Artículo 2, Número 17 (2019)

https://doi.org/10.46381/reic.v17i0.471

www.criminologia.net

ISSN: 1696-9219 


\section{Discusión}

Este artículo partía de dos objetivos, contribuir al conocimiento de las variables que afectan a la progresión penitenciaria, y explorar la capacidad de determinadas teorías criminológicas para explicar dicha progresión y dotar a este tipo de estudios de un marco teórico y explicativo de referencia.

En relación con el primer objetivo, de los resultados del modelo se puede destacar lo siguiente:

En primer lugar, se reafirma la importancia de algunas de las variables relacionadas con la trayectoria penitenciaria que la literatura previa había destacado. Así pues, se confirma que el hecho de tener una revocación del tercer grado dificulta la progresión (Cid \& Tébar, 2014), siendo la duración de la condena también relevante. Los resultados también indican que tendrán más obstáculos aquellas personas que fueron condenadas por un delito violento. Este aspecto debería ser tratado con atención por la investigación futura, puesto que podría indicar un etiquetamiento de los individuos en base a su pasado o, por el contrario, podría ser consecuencia de que personas estén siendo condenadas a penas cortas por delitos violentos (ej: lesiones y violencia contra la pareja) las cuales sabemos que presentan mayores limitaciones para progresar debido a los tiempos de clasificación. Por otra parte, los resultados nos permiten observar que la mayoría de factores propuestos por los profesionales como obstáculos para la progresión (Ibàñez \& Cid, 2016) efectivamente parece que lo son, con la excepción de la desventaja, no mencionada por los profesionales, pero sí relevante en nuestro modelo.

En segundo lugar, y en la línea de lo propuesto por Blevins et al. (2010) apreciamos como la teoría de la tensión puede ser un marco conceptual adecuado para estudiar el cómo la vivencia del encarcelamiento puede afectar al comportamiento de los individuos que cumplen condena. Recientemente Rodríguez Menés, Larrauri Pijoan y Güerri Ferrández (2018) en un estudio en diversas prisiones catalanas concluían que las causas que explican la calidad de vida en prisión también pueden servirnos para explicar el mal comportamiento intramuros. Por su parte, los resultados del presente artículo muestran que algunas fuentes de

Revista Española de Investigación Criminológica

Artículo 2, Número 17 (2019)

https://doi.org/10.46381/reic.v17i0.471

www.criminologia.net

ISSN: 1696-9219 
tensión, y especialmente el hecho de ser victimizado, se relacionan con una menor probabilidad de progresar. También parecen jugar un papel fundamental la exposición a coerción mediante las sanciones y la percepción de la legitimidad en el trato con los profesionales, en la línea de lo propuesto por Colvin (2007). Esto es relevante puesto que las sanciones no solo serían una medida para garantizar el orden interno de los centros, sino que podrían tener serias consecuencias para el futuro de los individuos en tanto parece que bloquean las posibilidades de progresar, poniendo el foco en su uso y en el trato del personal hacia los internos. En relación con lo anterior, este trabajo también ha mostrado cómo algunos mecanismos de afrontamiento de la tensión, y en concreto la autoestima y el apoyo social son elementos que favorecen la salida progresiva, aportando nueva evidencia a un campo poco explorado que vincula la falta de mecanismos y recursos de afrontamiento con un peor comportamiento en prisión (Rocheleau, 2014) y con peores perspectivas de reinserción en el futuro (LaCourse, Listwan, Reid \& Hartman, 2018). Concretamente los resultados han mostrado la relevancia del apoyo informal (a través de las familias) e institucional (mediante los profesionales) para el correcto desarrollo de la condena. Aunque la importancia del apoyo familiar ya ha sido anteriormente destacada en nuestro contexto cómo un elemento que favorece la reinserción (Cid \& Martí, 2012; Ibàñez \& Pedrosa, 2018; Martí \& Cid, 2015), no existe demasiada evidencia previa sobre la importancia del apoyo institucional sobre los individuos, por lo que este trabajo aporta nueva evidencia al respecto.

Por último, este trabajo también ha mostrado la importancia de la desventaja previa como elemento que no solo marca a las personas en su vida social fuera de la cárcel, sino que también tiene efectos en el desarrollo de la condena. Los resultados indican que las personas reincidentes, aquellas con un historial de consumo y aquellas con una trayectoria de desventaja laboral tendrán más obstáculos hacia la progresión penitenciaria. Si conceptualizamos los antecedentes como una forma de desventaja, podemos defender que la categoría "reincidente" en muchas ocasiones esconde bajo sí una trayectoria de desventaja y exclusión social más amplia. Esto contraviene la concepción de que únicamente se toma en cuenta la realidad de la pena que se cumple -concepción que como hemos visto han aceptado e integrado los profesionales- $y$, en cambio, nos dice que el pasado importa, y no

Revista Española de Investigación Criminológica

Artículo 2, Número 17 (2019)

https://doi.org/10.46381/reic.v17i0.471

www.criminologia.net

ISSN: 1696-9219 
precisamente en un sentido positivo. Esta desventaja previa puede ser relevante puesto que estos individuos llegarían a la cárcel con menos apoyo, recursos y capital social, con lo que quedarían más expuestos a los efectos negativos de la condena, haciendo que sean más vulnerables ante procesos de etiquetamiento.

En referencia al segundo objetivo, los resultados del modelo de regresión logística permiten afirmar que efectivamente hay variables relacionadas con la experiencia de la condena, el apoyo social y la desventaja, que permiten explicar porqué algunas personas tienen más dificultades que otras a la hora de progresar. Así lo demuestra el hecho de que incluir este tipo de variables hace que el ajuste y la capacidad explicativa del modelo mejoren respecto de tener en cuenta únicamente las variables identificadas por la literatura previa. Estas variables, además, ofrecen una explicación plausible que nos permite interpretar algunas variables que hasta ahora se han tratado como neutras o simplemente ligadas a la práctica penitenciaria, mediante una explicación teórica que nos permita entender por qué éstas están afectando a la progresión durante la condena. Por ejemplo, presentar las sanciones no únicamente como una respuesta al mal comportamiento de los internos, sino como un mecanismo de coerción, nos puede ayudar a entender por qué aquellos individuos más sancionados sufrirán mayor sensación de ilegitimidad, y podrán desarrollar sentimientos negativos que los lleven a desvincularse de la institución y a ser excluidos, hipótesis que deberá ser explorada por la investigación futura.

En resumen, al inicio empezábamos con una pregunta: ¿Quién se queda atrás en nuestro sistema penitenciario? Los resultados de esta investigación son un primer paso para tratar de abordar dicha cuestión, y lo que es más importante, dotarla de un marco explicativo de referencia. Los resultados han permitido afirmar que las personas más expuestas a los efectos negativos de la pena, aquellas que cuentan con menos apoyo, y aquellas con una trayectoria de desventaja, son las que se quedan atrás, o quizá las que el sistema deja atrás. Entre todos debemos plantearnos cómo hacer que la prisión sea una oportunidad para romper la espiral de exclusión en la que viven algunas personas, y no el inicio o continuación de dicha espiral. Esto se encuentra estrechamente ligado con la importancia de una salida progresiva como mecanismo para la reinserción, pero va más allá, y resulta necesario dotar

\section{Revista Española de Investigación Criminológica}

Artículo 2, Número 17 (2019)

https://doi.org/10.46381/reic.v17i0.471

www.criminologia.net

ISSN: 1696-9219 
a las personas que salen de prisión de los recursos y las oportunidades que necesitan. Quizá esto requiera un cambio de paradigma, una prisión orientada a dar apoyo y no a coercionar, una prisión que no excluya sino que brinde oportunidades.

\section{Implicaciones}

Esta investigación presenta importantes implicaciones, tanto a nivel práctico como de políticas en materia penitenciaria:

A nivel práctico, en primer lugar, es necesario eliminar aquellos obstáculos legales que aún dificultan la progresión penitenciaria para muchas personas, puesto que parece contraintuitivo que un sistema que debería estar basado en la reinserción y comportamiento del interno termine viéndose subyugado por factores como el tiempo de condena, por lo que debería apostarse por unos requisitos más flexibles. Quizá lo que estos resultados enmascaran es la realidad de que la prisión no es la medida adecuada para los delitos leves y las penas cortas, puesto que se observa cómo el propio sistema de progresión no está pensado para ellas. Especialmente destacan las grandes dificultades con las que se encuentran las personas extranjeras para progresar. Aunque haya cierta tendencia a presentar esto como una consecuencia administrativa - y neutra- asociada a la situación legal de la persona, esto no deja de constituir una discriminación inaceptable respecto al trato que nuestras instituciones dan a las personas extranjeras, viéndose estas excluidas de los mecanismos de progresión. Debemos preguntarnos cómo podemos esperar que estas personas cumplan y se involucren en su proceso de rehabilitación si sistemáticamente las excluimos de los mecanismos de liberación anticipada con independencia de su comportamiento o seguimiento del tratamiento.

Por otra parte, se defiende un uso limitado de la pena de prisión para evitar los males que esta comporta, y cuando esta sea inevitable, que se ejecute de la manera menos coercitiva posible, se atienda a las necesidades y el bienestar de las personas que cumplen condena, estos sean tratados de manera legítima y, en general, se trate de que el encarcelamiento afecte lo menos posible a la persona tanto a nivel individual como social. A su vez, este

Revista Española de Investigación Criminológica

Artículo 2, Número 17 (2019)

https://doi.org/10.46381/reic.v17i0.471

www.criminologia.net

ISSN: 1696-9219 
encarcelamiento no coercitivo debería prestar apoyo a las personas que cumplan condena, especialmente a través de una figura de referencia que se preocupe, respete y ayude a la persona encarcelada. Algunas medidas en este sentido podrían ser limitar el uso de las sanciones, apostando por la resolución alternativa de conflictos, favorecer el contacto con el exterior, y apostar por un tratamiento menos centrado en la figura del programa y más en la del profesional que lo imparte.

Por último, estas medidas, especialmente el apoyo institucional, deben ir dirigidos a aquellas personas más vulnerables y con más probabilidad de sufrir los efectos de la tensión, aquellas personas con menos recursos y apoyo, peor estado emocional, y que tengan trayectorias de desventaja y exclusión. Un sistema penitenciario humano y efectivo será aquel que trate de dar oportunidades a esas personas ya olvidadas, y no uno que contribuya a etiquetarlas y excluirlas aún más.

Evidentemente estas medidas deberán ir acompañadas de políticas públicas que sustenten este modelo de reinserción, den recursos y oportunidades a las personas mientras cumplen condena y una vez que estas retornen a la comunidad, destacando especialmente aquellas medidas dirigidas a la inserción laboral de aquellas personas con menos formación y que se hayan visto excluidas del mercado de trabajo. Sólo a través de este tipo de medidas podremos acercarnos y trabajar para que la prisión sea una oportunidad y no un obstáculo para la reinserción.

\section{Revista Española de Investigación Criminológica}

Artículo 2, Número 17 (2019)

https://doi.org/10.46381/reic.v17i0.471

www.criminologia.net

ISSN: 1696-9219 


\section{Referencias}

Agnew, R. (1992). Foundation for a general strain theory of crime and delinquency. Criminology, 30(1), 47-88. doi: 10.1111/j.1745-9125.1992.tb01093.x

Agnew, R. (2001). Building on the foundation of general strain theory: Specifying the types of strain most likely to lead to crime and delinquency. Journal of research in crime and delinquency, 38(4), 319-361. doi: 10.1177/0022427801038004001

Agnew, R. (2006). Pressured into crime: An overview of general strain theory. New York: Oxford

Agnew, R. (2009). General strain theory: Current status and directions for further research. En Cullen, F. T., Wright, J.P., \& Blevins, K.R. (Eds.), Taking stock: The status of criminological theory. New Brunswick, $\mathrm{Nj}$ : Transaction

Blevins, K. R., Listwan, S. J., Cullen, F. T., \& Jonson, C. L. (2010). A general strain theory of prison violence and misconduct: An integrated model of inmate behavior. Journal of Contemporary Criminal Justice, 26(2), 148-166. doi: $10.1177 / 1043986209359369$

Broidy, L., \& Agnew, R. (1997). Gender and crime: A general strain theory perspective. Journal of research in crime and delinquency, 34(3), 275-306. doi: $10.1177 / 0022427897034003001$

Capdevila, M. C., Blanch, M. S., Ferrer, M. P., Pueyo, A. A., Framis, B. F., Comas, N. L, Garrigós, A. B., Boldú, A. P., Batlle, A. M., \& Mora, J. E. (2015). Taxa de reincidència penitenciària 2014. Barcelona: Centre d'Estudis Jurídics i Formació Especialitzada.

Capdevila, M. C., Parés, R. G., Ferrer, M. P., Luque, E. R., \& Torrecillas, M. M. (2006). La classificació inicial en règim obert. Barcelona: Centre d'Estudis Jurídics i Formació Especialitzada.

Cid, J. (2005). The penitentiary system in Spain: The use of imprisonment, living conditions and rehabilitation. Punishment \& Society, 7(2), 147-166. doi: $10.1177 / 1462474505050439$

Cid, J. (2009). Is imprisonment criminogenic? A comparative study of recidivism rates between prison and suspended prison sanctions. European Journal of Criminology, 6(6), 459-480. doi: 10.1177/1477370809341128

Cid, J., \& Martí, J. (2012). Turning points and returning points: Understanding the role of family ties in the process of desistance. European Journal of Criminology, 9(6), 603620. doi: $10.1177 / 1477370812453102$

Cid, J., \& Tébar, B. (2010). Libertad condicional y delincuentes de alto riesgo. Revista española de investigación criminológica, 8(3), 1-23.

Revista Española de Investigación Criminológica

Artículo 2, Número 17 (2019)

https://doi.org/10.46381/reic.v17i0.471

www.criminologia.net

ISSN: 1696-9219 
Cid, J., \& Tébar, B. (2014). La revocación del régimen abierto: ¿Una práctica legítima? (Recall from open regime and legitimacy). Cuadernos de política criminal, (114), 199-232.

Colvin, M. (2007). Applying differential coercion and social support theory to prison organizations: The case of the penitentiary of New Mexico. The Prison Journal, 87(3), 367-387. doi: 10.1177/0032885507304774

Colvin, M., Cullen, F. T., \& VanderVen, T. (2002). Coercion, social support, and crime: An emerging theoretical consensus. Criminology, 40(1), 19-42. doi: 10.1111/j.17459125.2002.tb00948.x

Cullen, F. T. (1994). Social support as an organizing concept for criminology: Presidential address to the Academy of Criminal Justice Sciences. Justice Quarterly, 11(4), 527559. doi: 10.1080/07418829400092421

Férez-Mangas, D., \& Andrés-Pueyo, A. (2015). Predicción y prevención del quebrantamiento de los permisos penitenciarios. Revista Española de Investigación Criminológica, (13), 7-28.

Ibàñez, A., \& Cid, J. (2016). La reinserción de las personas que finalizan condena en régimen ordinario. Barcelona: Centre d'Estudis Jurídics i Formació Especialitzada

Ibàñez, A., \& Pedrosa, A. (2018). El paper de les famílies en la reinserció de les persones que surten de la presó. Barcelona: Centre d'Estudis Jurídics i Formació Especialitzada

Kort-Butler, L. A. (2010). Experienced and vicarious victimization: Do social support and self-esteem prevent delinquent responses? Journal of Criminal Justice, 38(4), 496505. doi:10.1016/j.jcrimjus.2010.04.019

Laub, J. H., \& Sampson, R. J. (1993). Turning points in the life course: Why change matters to the study of crime. Criminology, 31(3), 301-325. doi: 10.1111/j.17459125.1993.tb01132.x

Link, B. G., Cullen, F. T., Struening, E., Shrout, P. E., \& Dohrenwend, B. P. (1989). A modified labeling theory approach to mental disorders: An empirical assessment. American sociological review, 400-423. doi: 10.2307/2095613

LaCourse, A., Listwan, S. J., Reid, S., \& Hartman, J. L. (2019). Recidivism and Reentry: The Role of Individual Coping Styles. Crime \& Delinquency. doi: $10.1177 / 0011128718790497$

Listwan, S. J., Sullivan, C. J., Agnew, R., Cullen, F. T., \& Colvin, M. (2013). The pains of imprisonment revisited: The impact of strain on inmate recidivism. Justice Quarterly, 30(1), 144-168. doi: 10.1080/07418825.2011.597772

Luque, E. R., Ferrer, M. P., \& Capdevila, M. C. (2005) La reincidència penitenciària a Catalunya. Barcelona: Centre d’Estudis Jurídics i Formació Especialitzada.Martí, J.,

Revista Española de Investigación Criminológica

Artículo 2, Número 17 (2019)

https://doi.org/10.46381/reic.v17i0.471

www.criminologia.net

ISSN: 1696-9219 
\& Cid, J. (2015). Encarcelamiento, lazos familiares y reincidencia. Explorando los límites del familismo. Revista Internacional de Sociología, 73(1), 002.

Nagin, D. S., Cullen, F. T., \& Jonson, C. L. (2009). Imprisonment and reoffending. Crime and justice, 38(1), 115-200. doi: 10.1086/599202

Nilsson, A., Bäckman, O., \& Estrada, F. (2013). Involvement in crime, individual resources and structural constraints: Processes of cumulative (dis) advantage in a Stockholm birth cohort. British Journal of Criminology, 53(2), 297-318. doi: 10.1093/bjc/azs068

Rocheleau, A. M. (2014). Prisoners' coping skills and involvement in serious prison misconduct. Victims \& Offenders, 9(2), 149-177. doi: $10.1080 / 15564886.2013 .866916$

Rodríguez Menés, J., Larrauri Pijoan, E., \& Güerri Ferrández, C. (2018). Percepción de la calidad de vida en prisión. La importancia de una buena organización y un trato digno. Revista Internacional de Sociología, 76(2), 098. doi: 10.3989/ris.2018.76.2.16.159

Sampson, R., \& Laub, J. (1997). A life-course theory of cumulative disadvantage and the stability of delinquency. En T. Thornberry (ed.), Developmental theories of crime and delinquency. New Brunswick: Transaction publishers.

Steiner, B., Butler, H. D., \& Ellison, J. M. (2014). Causes and correlates of prison inmate misconduct: A systematic review of the evidence. Journal of Criminal Justice, 42(6), 462-470. doi: 10.1016/j.jcrimjus.2014.08.001

Sykes, G. (1958). The society of captives: A study of a maximum security prison. Princeton. NJ: Princeton University Press

Tébar, B. (2004). Variables de la concessió de la llibertat condicional a Catalunya. Barcelona: Centre d'Estudis Jurídics i Formació Especialitzada.

Visher, C. A., \& O'Connell, D. J. (2012). Incarceration and inmates' self perceptions about returning home. Journal of Criminal Justice, 40(5), 386-393. doi: 10.1016/j.jcrimjus.2012.06.007 


\section{Agradecimientos}

Este trabajo ha estado realizado en el marco del programa de doctorado en Derecho de la Universitat Autònoma de Barcelona. Quiero agradecer la revisión y comentarios de José Cid en versiones anteriores del manuscrito, a la SEIC por considerar que era merecedor de un premio, y especialmente a compañeros y amigos que me apoyan y con quiénes comparto precariedad, pero sobre todo buenos momentos. Este proyecto no cuenta con financiación de ningún organismo público o privado.

Albert Pedrosa Bou es graduado en Criminología por la Universitat Autònoma de Barcelona y Máster en Criminología y Ejecución Penal por la Universitat Pompeu Fabra. Actualmente se encuentra vinculado al grupo de investigación "Desistimiento del Delito y Políticas de Reinserción" de la Universitat Autònoma de Barcelona como investigador predoctoral (Beca FPI 2015). Sus temas de interés son el estudio del uso de la prisión y la situación de las familias de las personas encarceladas.

iD https://orcid.org/0000-0002-3533-7282 